\title{
Software Process Improvement: Influence Factors-Problem, Cause, Solution
}

\author{
${ }^{1}$ Pankaj Kumar, ${ }^{2}$ Monika Dhariwal, ${ }^{3}$ Suresh Kumar Yadav, ${ }^{4}$ Gajraj Singh \\ ${ }^{1,3,4}$ Department of Mathematics Ramjas College, University of Delhi, ${ }^{2}$ MITS Lakshmangarh,Rajasthan
}

\begin{abstract}
Software Process Improvement (SPI) is a systematic approach and continuous improvement of software development organization's ability to produce and deliver quality software with in time and budget constraints [1]. This paper describes the principle of the software process improvement and the factors which influence on the Software Process Improvement on the basis of survey and give solutions.
\end{abstract}

Keywords: Software Process Improvement, Software Process Improvement Model, Software Quality.

\section{Introduction}

Software process improvement is an approach for the process improvement. A crisis came in software engineering around 1965-1985. This time the failure of the project was increased. The cause of the software crises were linked to the overall complexity of the software process and the relative immaturity of software engineering as a profession. The crises manifested it in several ways:

- Project running over budget

- Project running over time

- Software was low quality

- Software often did not meet requirements

- Project was unmanageable and code difficult to maintain [2].

Through this paper researcher tells about the factors which is the cause of this crises and explain the factors of software process improvement. A lot of problem had come in the past. The solution of these problems comes time to time. A lot of models have to come in existence. Process improvement is the technique of improving the quality of product as well as organization. Many maturity models are currently used in process improvement. The ultimate aim is to develop a model which would be useful in practice for software development companies. During the 1990s there has been a movement in the software development community towards the improvement of the software process as a means to improve the quality of software. Small software development companies are important to the Indian economy, and therefore, should keep up-to-date with global developments. Software process: Software process is the task or activities used for produce a software product. It is not necessary its output is software. A process can generate the other process. It is a set of activities, methods, practices, and transformations that people use to develop and maintain software and its associated products [3]. A lot of factor plays important role in software process. Project should be with in time under budget and better quality for long term business. In this paper section II describe the meaning of Software Process Improvement and section III define the cause of problem facing in the project. Section IV suggests the solution for these problems.

\section{Software Process Improvement}

Process is a set of activity which gives an output it may be a process or product. Output in the sense of software is said to software process which output should be software. Software process improvement is a set of activities by which an organization can get a high level of maturity in the product and get a high level product [5]. Process improvement is a change in the process to get a better output rather than original output. There are some activities for this;

- Identify the current process.

- Identify the current status of the organization.

- Identify the objects and their attributes.

- Identify the operation on the objects.

- Identify the relation object attributes and operations.

- Identify the strength and weakness of the organization.

- Analyze where the changes are needed.

- What will be the effect of these changes?

- Understand these changes that how much they are affected. 
A simple software process improvement life cycle is presented in the fig 1 below

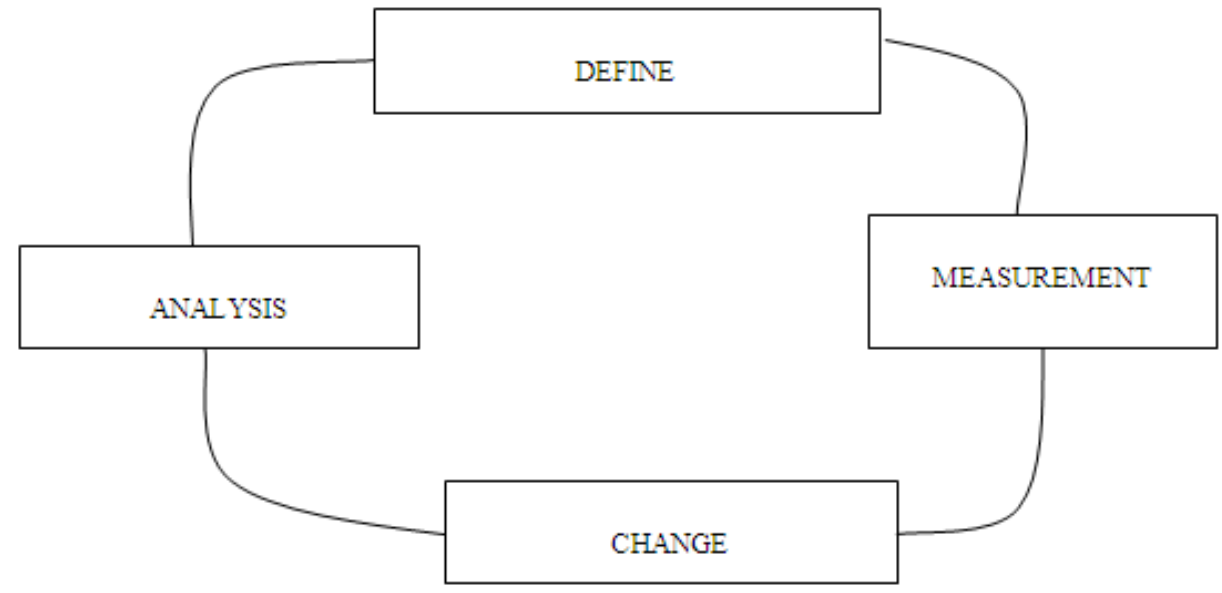

Fig 1 Software process improvement life cycle

III. Problems In Software projects

There are mainly three problems to be solved in the software project.

- Projects have no good quality

- Project does not deliver with in time

- Project goes over budget

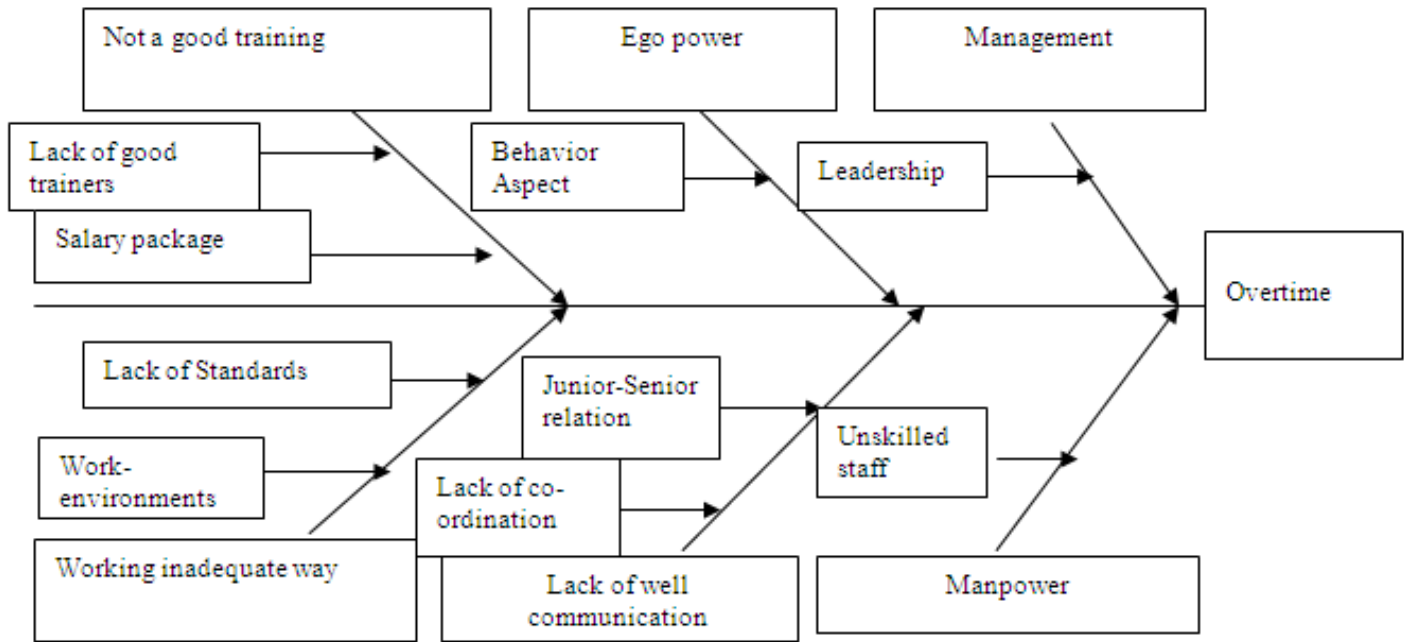

Fig 2: fish bone diagram for overtime the projects

Every problem has some causes, which are presented by the fishbone diagram. For example a project that does not get a good quality. Beyond these problems some causes are there. Management is one the cause of this problem but this is also a problem again beyond this problem there are a lot of reasons not only a good management. There may not be good communication between the manager and employee. Project manager are not sincere and he could not understand the problem and handle the problems. He could not present the problem in adequate way to the senior management. Senior management don't hear the problems of employees.

Another cause of the problem of bad quality is misunderstanding. If organization cannot do a good calculation of the project size at the time of deal with the customer. Then problem creates because requirement cannot change after the completion of the deal. Organization wants that they should complete the project under budget. At this situation it is not possible that project gets the good quality. Another cause of the bad quality problem is manpower. If organizations do not find suitable employees in the project then project will be either late or not a good quality. Employees should be well trained. They should be aware to new technologies. If they don't know the new technologies or don't aware to the technologies. Then they can't perform well in their module. 


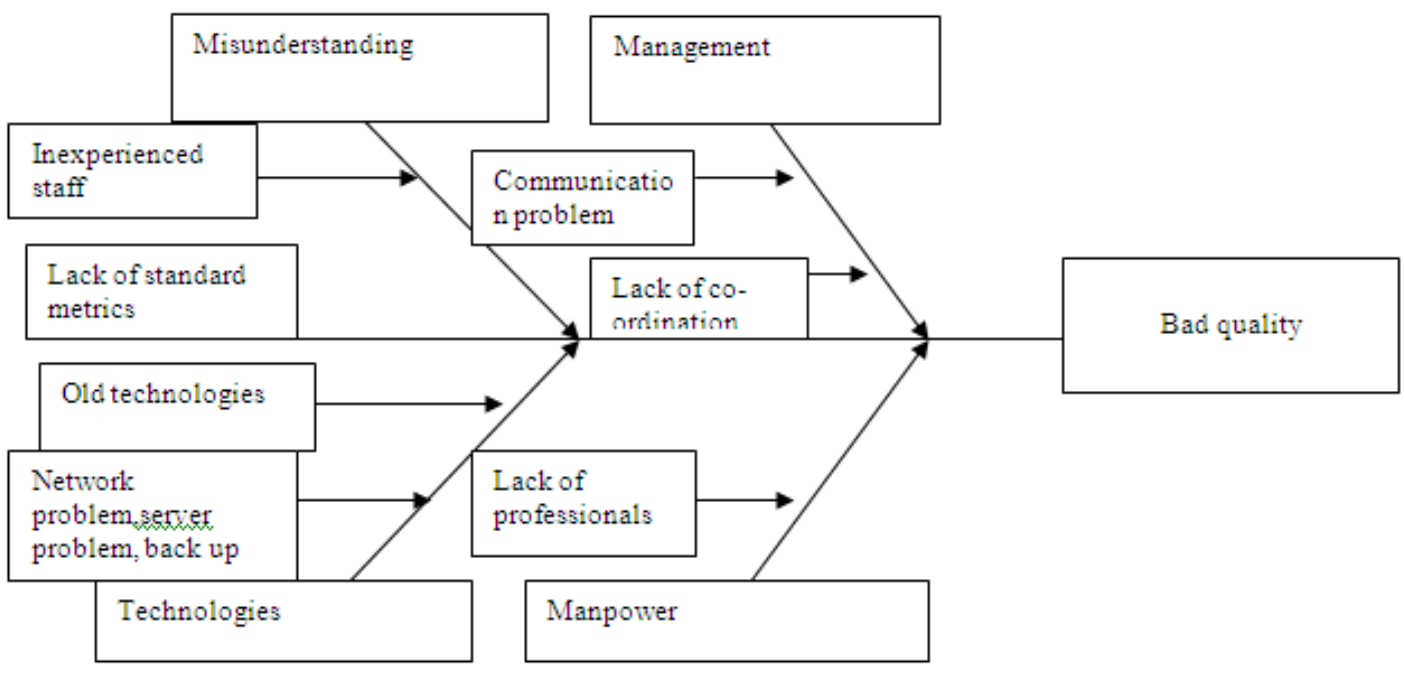

Fig 3: Fish Bone Diagram of problem Bad Quality

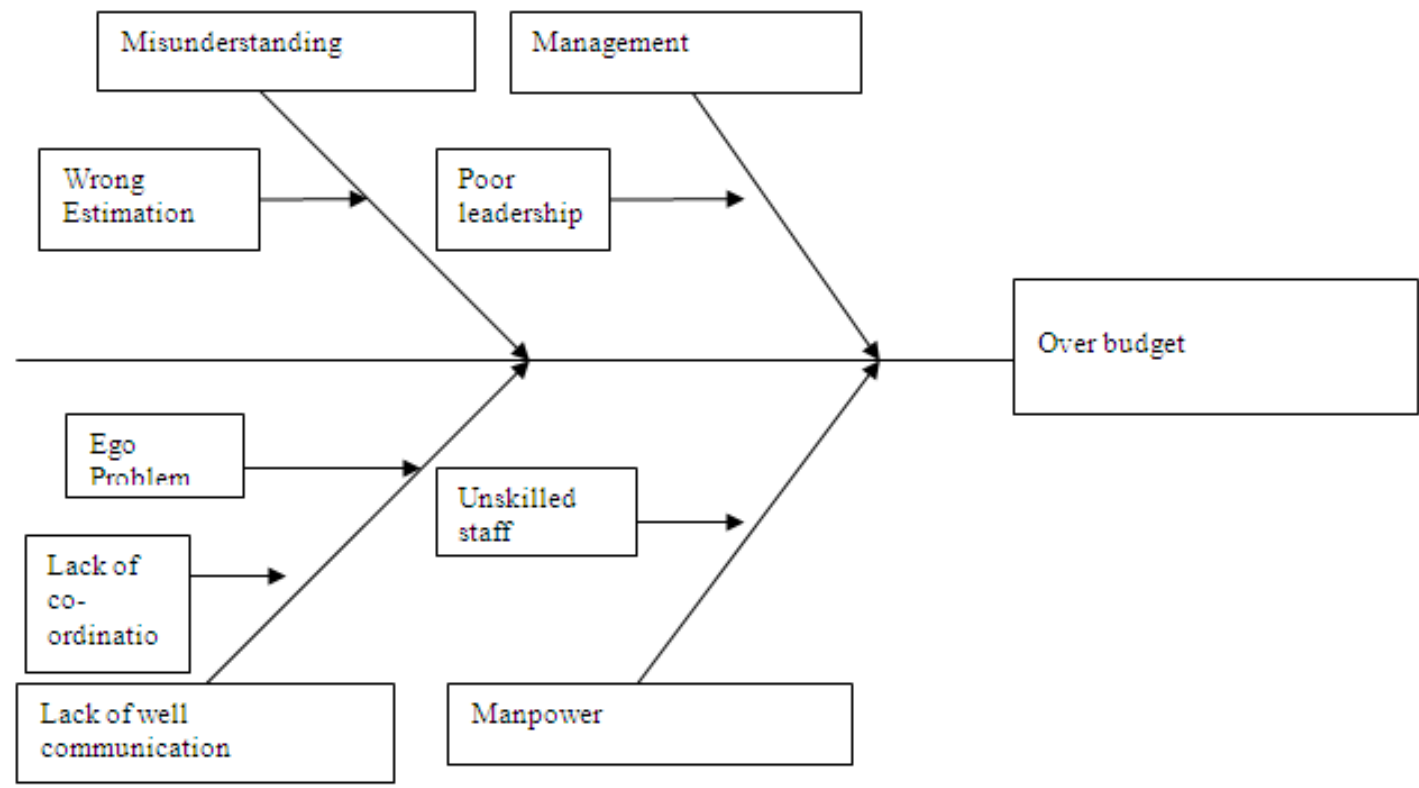

Fig 4: fishbone diagram for the over budget in project

Second problem is over budget. Project sometime goes to over budget. The main cause this problem is misunderstanding between the customer and organization. At that time they have done mistake to calculate the project. Than at this situation project goes to over budget. Third problem is over time. The project does not complete with in time which is deadline given by the customer. The main problem is not provided a good training to the employee. They don't have a good knowledge about the subject what they are going to do. So they can't perform well and the projects cross the deadline.

\section{Reason of problem face in projects:}

1) Lack of management

2) Lack of well communication

3) Lack of well communication between customer and organization

4) Technical problem and lack of resources

5) Lack of man power

6) Not provided a good training

7) Ego power

8) Lack of standards [6] 
1) Lack of management: This is a good point of view for unsuccessful project. If there is no good management in the organization. This is harmful for the project. Project manager don't so well qualified then he cannot hold the team and cannot inspire the team. Senior management does not hear the problem or ignore them.

2) Lack of co-ordination: Well-known means suppose a man which is perfect in one module of a project. But management gives him other responsibility in the project then this person cannot perform well as well as he can do in his module. If every member in the team know its work very well with interest. Then output may be come in a better way and better quality.

3) Well communication between customer and organization: Well communication means when organization deals with the customer. They must specify the entire requirement according to the customer. Calculate everything according to the size, budget, and time. If organization fails to right estimation with the deal in customer then project can't get a good quality. Because organizations don't want that they go over budget. So they want to complete project according the customer's budget. So they can't give a good quality in the project.

4) Lack of man power: Lack of manpower or good manpower is also a reason for unsuccessful project. For example a project needs 100 men but due to some reason you have only 50 men. Reason may be anything like you has no such budget for project, or you cannot arrange so much manpower but you have taken a big project. At this condition project cannot complete on time. One more reason organization have no good manpower that is if one person works on a modules but he leave after some day at this situation also a better output has not come with in time.

5) Not a good training: Organization should provide a good training to member of team. Without good training they can't perform so well. They can't get higher level of maturity without training. In the training organization should give the good knowledge and tell them what the organization want.

6) Ego problem: A well communication have establish in member of companies. If members have ego power and not co-operate with other then project will not go in a right way.

Table 1: Decision table for cause and effect on software project

\begin{tabular}{|l|l|l|l|}
\hline CAUSE & 1 & 2 & 3 \\
\hline Lack of management & Y & Y & Y \\
\hline Misunderstanding & Y & & Y \\
\hline Not provide good training & & Y & \\
\hline Technologies & Y & & \\
\hline Not well Communication & & & Y \\
\hline Inadequate work & & Y & \\
\hline Lack of well communication & & Y & Y \\
\hline Manpower & Y & Y & Y \\
\hline Ego power & & Y & \\
\hline EFFECT & & & \\
\hline Bad quality & Y & & \\
\hline Over budget & & & Y \\
\hline Over time & & Y & \\
\hline
\end{tabular}

Following table describe the criticality of project on the category of 5 scales:

1. Initial: this scale is not so harmful.

2. Minor: this scale shows the effects on the project are minor that can be modified in time being.

3. Moderate: this scale shows the effects on the project that can be become cause of crashing the project but can be modified with some effort.

4. Major: this scale shows the effects on the project that can be cause of became cause of the crashing the project and big efforts are needed to resolve the problem.

5. Critical: this scale is most dangerous scale and become the cause of failure of project.

\begin{tabular}{|l|l|l|}
\hline & Cause of problems & Category out of 5 scale \\
\hline 1 & Lack of management & 4 \\
\hline 2 & Lack of well communication & 3 \\
\hline 3 & Lack of well communication between costumer and organization & 3 \\
\hline 4 & Technical problem and lack of resources & 4 \\
\hline 5 & Lack of man power & 3 \\
\hline 6 & Not provided a good training & 3 \\
\hline 7 & Ego problem & 2 \\
\hline 8 & Working inadequate way & 2 \\
\hline
\end{tabular}

\section{Some Steps for the Process Improvement}

A) Aware for Organization Culture: Always be aware to the organization culture. Know about the things which are important for the organization. What current technology is in the picture? What should come in future? Develop your self-according to them. 
B) Consistence should be there: Consistency means discussion with all the members of the team. Before starting the work, a formal meeting should be conducted, to know whether all things are complete. No requirement is left. Divide the work according to the ability of suitable person. Commitment also plays a very important role in this, because the person who takes the responsibility of a particular work should complete the work within time and respect the seniors.

C) Make Target and Keep in mind: When project comes to the company. Discuss with the customer very frankly. According to the requirements, estimates the size of the project. And make the target what we are going to do in future. All requirements should be clear otherwise some problem may be faced in the middle of project. Then work should be done according to the plan.

D) Put an option in the Process: When people of an organization works on a project. They keep a copy of that work. If there is any problem in that project at any stage for some reason. Then work has to be done again from the baseline. For example, if a project has to be done in .NET but some problem is faced in doing that project in .NET, then, use the alternate process for completing the project.

E) Communication between the team member and Senior Management: A good communication should be established among the team management and senior management. An employee can discuss with the seniors directly. They can give the new ideas about the problems. Seniors should listen to these ideas and should give a proper response to these ideas. They should promote people not only on the basis of experience but also on the basis of their knowledge.

F) Treat Process Improvement like a Project: Be serious for the process improvement. Define the problems for the process, then make a suitable document for these, make the plan according to them, then implement it in a right way to improve the process. There should be an experienced manager and a well-qualified team for the process improvement.

G) Make changes according to the Priority: Process improvement should not be done just once. It should be given priority. Priority should be according to the various standards. Such as, it should be done with CMM or SPICE or ISO standard.

H) Make a profile of the Company: First make the profile of the organization. Collect the strength and weakness of the company. It should be done by review. Everything has a strong point and weak point. Make a profile for this. And concentrate on how to remove the weaknesses.

I) Introduce a Software Process Engineering Group (SEPG) to organization: There should be groups in the organization which is well qualified and can inspect the process. Shortcomings are to be told to the members and provide the advice to improve them. This group should be well qualified and certified by the Software Engineering Institute (SEI). This group should take a lot of responsibility for the organization. So member of the team should be highly qualified.

J) Have Patience: Some time process may be slow and costs are over budget [4]. So it is important to have patience. Sometime this process takes a long time.

K) Reuse the previous Result: Software process improvement is a methodology or a model. After successful of this methodology next project can reuse this result.

\section{Conclusion and Future Work}

This paper tells the software process improvement and the factors why organization needs for process improvement. Organization does not get the good quality and maturity product because of a lot of problem existing in its internal. Some technique for software process improvement in this paper may be a solution of these problems. Software process improvement is a broad area. Every company wants to improve their process and get a high level product. A lot of models come in to the picture like as CAPABLITY MATURITY MODEL (CMM), IDEAL, BOOTSTRAP, SPICE, SIX SIGMA and a lot of. New technologies come time to time. Organization should be adopting itself according them.

\section{Reference}

[1]. Jesper Arent, Jacob Nørbjerg "Software Process Improvement as Organizational Knowledge Creation: A Multiple Case Analysis," Proceedingsof the 33rd Hawaii International Conference onSystem Sciences -2000

[2]. The American Corporation Today<http://www.questia.com/googleScholar.qst?docId=80352426>

[3]. Laurie Honour Werth, "Lecture Notes on Software Process Improvement", document number CMU/SEI-93-EM-8, copyright by Carnegie Mellon University, "Introduction to Software Process Improvement", 1993.

[4]. Mohd. Hairul Nizam, Md. Nasir, Rodina Ahmad, Noor Hafizah Hassan, "Resistance Factors in the Implementation of Software Process Improvement Project", Volume: 4, pp. 1-10, 26-28 Aug. 2008.

[5]. Anthony Finkelstein, "Software Process Immaturity Model", Software engineering notes 17,vol. no. 4,Oct 1992 pp 22-23.

[6]. Zhedan Pan, Hyuncheol Park, Jongmoon Baik, HojinChoi, "A Six Sigma Framework for Software Process Improvements and its Implementation", Proceedings of the 14th Asia-Pacific Software Engineering Conference, ISBN ISSN: 1530-1362, 0-7695-30575, pp. 446-453 2007. 